T. Kataoka

Nagoya Math. J.

Vol. 77 (1980), 25-31

\title{
ON THE INTEGER RING OF THE COMPOSITUM OF ALGEBRAIC NUMBER FIELDS
}

\author{
TOSHITAKA KATAOKA
}

\section{§1. Statement of the results}

Let $k$ be an algebraic number field of finite degree. For a finite extension $L / k$ we denote by $\mathfrak{D}_{L / k}$ the different of $L / k$, and by $\mathfrak{O}_{L}$ the integer ring of $L$. Let $K_{1}$ and $K_{2}$ be finite extensions of $k$. It is known that we have $\mathfrak{O}_{K_{1} K_{2}}=\mathfrak{D}_{K_{1}} \mathfrak{O}_{K_{2}}$ if $K_{1}$ and $K_{2}$ are linearly disjoint over $k$ and $\mathfrak{D}_{K_{1} K_{2} / k}$ $=\mathfrak{D}_{K_{1} / k} \mathfrak{Q}_{K_{2} / k}$ holds (see Shimura [2], 1.2).

In this paper we compute the conductor of $\mathfrak{O}_{K_{1}} \mathfrak{O}_{K_{2}}$ with respect to $\mathfrak{O}_{K_{1} K_{2}}$ and the module index of $\mathfrak{S}_{K_{1} K_{2}}$ and $\mathfrak{O}_{K_{1}} \mathfrak{O}_{K_{2}}$ in terms of relevant differents and "Elements". We note that the conductor of $\mathfrak{D}_{K_{1}} \mathfrak{D}_{K_{2}}$ with respect to $O_{K_{1} K_{2}}$ is the largest ideal of $\mathfrak{N}_{K_{1} K_{2}}$ which is contained in $\mathfrak{D}_{K_{1}} \mathfrak{O}_{K_{2}}$. For a Dedekind domain $R$ whose quotient field is $L$ and $R$-lattices $M, N$ of the same finite dimensional vector space over $L$, we denote by $[M: N]_{R}$ the module index of $M$ and $N$. We note that the index [M:N] is the absolute norm of $[M: N]_{R}$ if $L$ is a number field and $R$ is its integer ring. For general properties of module indices we refer to Frölich [1]. For a finite extension $L / K$ of algebraic number fields of finite degree and an embedding $\sigma$ of $L$ over $K$, we denote by $e_{\sigma}$ the element with respect to $\sigma$. We recall that $e_{\sigma}$ is the ideal generated by $x-x^{\sigma}, x \in \mathcal{D}_{L}$.

We state our results.

THEOREM. Let $k$ be an algebraic number field of finite degree, and $K_{1}$, $K_{2}$ its finite extensions. Then we have

(1) the conductor $\mathfrak{f}$ of $\mathfrak{O}_{K_{1}} \mathfrak{O}_{K_{2}}$ with respect to $\mathfrak{D}_{K_{1} K_{2}}$ is $\prod_{o \neq 1} e_{\sigma \mid K_{2}} \mathfrak{D}_{K_{1} K_{2} / K_{1}}^{-1}$, where $\sigma$ runs through all the non-trivial embeddings of $K_{1} K_{2}$ over $K_{1}$,

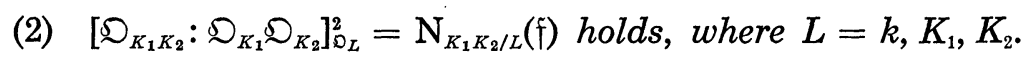

CoROLLARY. Let notations be as in Theorem. Then we have $\mathfrak{O}_{K_{1} K_{2}}=$ $\mathfrak{O}_{K_{1}} \mathcal{O}_{K_{2}}$ if and only if $\mathfrak{D}_{K_{1} K_{2} / K_{1}}=\prod_{\sigma \neq 1} \mathfrak{e}_{\sigma \mid K_{2}}$ holds.

Received October 2, 1978. 
We note that $\prod_{\sigma \neq 1} e_{\sigma \mid K_{2}}=\mathfrak{D}_{K_{2} / k}$ holds if $K_{1}$ and $K_{2}$ are linearly disjoint over $k$.

We shall give another description of the conductor and some examples in $\S 3$.

\section{§2. Proofs}

2.1. Proof of Theorem (1). Firstly we claim that there exists an element $z \in \mathfrak{D}_{K_{2}}$ for any prime ideal $\mathfrak{p}$ of $K_{2}$ such that

(i) $k(z)=K_{2}$,

(ii) the $\mathfrak{\beta}$-component of the conductor of $\mathfrak{D}_{K_{1}} \mathcal{O}_{K_{2}}$ is that of $\mathfrak{D}_{K_{1}}[z]$ for all prime ideals $\mathfrak{P}$ of $K_{1} K_{2}$ above $\mathfrak{p}$.

We take $z \in \mathfrak{D}_{K_{2}}$ which satisfies

(iii) $\operatorname{ord}_{\mathfrak{p}} f_{z}^{\prime}(z)=\operatorname{ord}_{\mathfrak{p}} \mathfrak{D}_{K_{2} / k}$, and $\operatorname{deg} f_{z}=\left[K_{2}: k\right]$.

Here $f_{z}$ is the minimal polynomial of $z$ over $k$. We show $z$ satisfies (ii). We recall that the conductor of $\mathfrak{D}_{k}[z]$ with respect to $\mathfrak{D}_{K_{2}}$ is $f_{z}^{\prime}(z) \mathfrak{D}_{K_{2} / k}^{-1}$, where $f_{z}^{\prime}$ is the derivative of $f_{z}$. We have

$$
\begin{aligned}
\mathfrak{D}_{K_{1}}[z] & =\mathfrak{D}_{K_{1}} \mathfrak{D}_{k}[z] \supset \mathfrak{D}_{K_{1}} f_{z}^{\prime}(z) \mathfrak{D}_{K_{2} / k}^{-1} \\
& =\mathfrak{D}_{K_{1}} \mathfrak{D}_{K_{2}} f_{z}^{\prime}(z) \mathfrak{D}_{K_{2} / k}^{-1} \supset \mathfrak{f} f_{z}^{\prime}(z) \mathfrak{D}_{K_{2} / k}^{-1} .
\end{aligned}
$$

Therefore the conductor of $\mathfrak{D}_{K_{1}}[z]$ contains $f f_{z}^{\prime}(z) \mathfrak{D}_{K_{2} / k}^{-1}$. Since ord $f_{p}^{\prime}(z) \mathfrak{D}_{K_{2} / k}^{-1}$ $=0$, we get the claim.

By the claim $\mathfrak{f}$ is the greatest common divisor of the conductors of $\mathfrak{D}_{K_{1}}[z]$, where $z$ satisfies (i) and is contained in $\mathfrak{D}_{K_{2}}$. The conductor of $\mathfrak{D}_{K_{1}}[z]$ with respect to $\mathfrak{D}_{K_{1} K_{2}}$ is $g_{z}^{\prime}(z) \mathfrak{D}_{K_{1} K_{2} / K_{1}}^{-1}$, where $z$ is an element of $\mathfrak{D}_{K_{2}}$ with (i) and $g_{z}$ is the minimal polynomial of $z$ over $K_{1}$. We show that

$$
\prod_{\sigma \neq 1} e_{\sigma \mid K_{2}}=\left(g_{z}^{\prime}(z): z \in \mathfrak{D}_{K_{2}}\right. \text { with (i)), }
$$

where $\sigma$ runs through all the non-trivial embeddings of $K_{1} K_{2}$ over $K_{1}$ into a finite Galois extension $L$ over $k$ containing $K_{1} K_{2}$. Let $\mathfrak{p}$ be a prime ideal of $K_{2}$. Let $z$ be an element of $\mathfrak{D}_{K_{2}}$ satisfying (iii). Then we have

$$
\operatorname{ord}_{\mathfrak{B}}\left(z-z^{\sigma}\right) \geq \operatorname{ord}_{\mathfrak{B}} \mathfrak{e}_{\sigma \mid K_{2}}
$$

for all the non-trivial embeddings $\sigma$ of $K_{1} K_{2}$ over $K_{1}$ into $L$ and all prime ideals $\mathfrak{P}$ of $L$ above $\mathfrak{p}$, and

$$
\sum_{\sigma \neq 1} \operatorname{ord}_{\mathfrak{\beta}}\left(z-z^{\sigma}\right)=\operatorname{ord}_{\mathfrak{B}} \mathfrak{D}_{K_{2} / k}=\sum_{\sigma \neq 1} \operatorname{ord}_{\Re} e_{\sigma}
$$

for all prime ideals $\mathfrak{P}$ of $L$ above $\mathfrak{p}$. Here the sums are taken over all 
the non-trivial embeddings $\sigma$ of $K_{2}$ over $k$ into $L$. Therefore we have

$$
\operatorname{ord}_{\mathfrak{B}}\left(z-z^{\sigma}\right)=\operatorname{ord}_{\mathfrak{B}} \mathrm{e}_{\sigma \mid K_{2}}
$$

for all non-trivial embeddings $\sigma$ of $K_{1} K_{2}$ over $K_{1}$ into $L$ and all prime ideals $\mathfrak{P}$ of $L$ above $\mathfrak{p}$. Using the decomposition $g_{z}^{\prime}(z)=\prod_{\sigma \neq 1}\left(z-z^{\sigma}\right)$, we have

$$
\operatorname{ord}_{\mathfrak{B}} g_{z}^{\prime}(z)=\operatorname{ord}_{\mathfrak{B}} \prod_{\sigma \neq 1} e_{\sigma \mid K_{2}}
$$

for all prime ideals $\mathfrak{P}$ of $L$ above $\mathfrak{p}$. Thus we proved the assertion. Hence we have (1).

2.2. Proof of Theorem (2). It suffices to prove the case $L=K_{1}$. For a $\mathfrak{O}_{K_{1}}$-lattice $M$ of $K_{1} K_{2}$ we denote by $M^{*}$ the dual module of $M$ with respect to $\operatorname{Tr}_{K_{1} K_{2} / K_{1}}$ We have

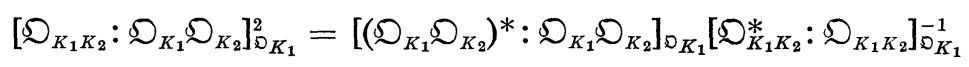

(see Fröhlich [1], Proposition 4, §3). Since $\mathfrak{D}_{K_{1} K_{2}}^{*}=\mathfrak{D}_{K_{1} K_{2} / K_{1}}^{-1}$,

$$
\begin{aligned}
{\left[\mathfrak{D}_{K_{1} K_{2}}^{*}: \mathfrak{D}_{K_{1} K_{2}}\right]_{\mathfrak{O}_{K_{1}}} } & =\left[\mathfrak{D}_{K_{1} K_{2} / K_{1}}^{-1}: \mathfrak{D}_{K_{1} K_{2}}\right]_{\mathfrak{O}_{K_{1}}} \\
& =\mathrm{N}_{K_{1} K_{2} / K_{1}}\left(\mathfrak{D}_{K_{1} K_{2} / K_{1}}\right)
\end{aligned}
$$

holds.

From now on we compute the module index $\left[\left(\mathfrak{D}_{K_{1}} \mathfrak{D}_{K_{2}}\right)^{*}: \mathfrak{D}_{K_{1}} \mathfrak{O}_{K_{2}}\right]_{\mathfrak{O}_{K_{1}}}$.

Lemma 1. Let $S$ be a finite set of prime ideals of $K_{1} K_{2}$. Let $\mathfrak{p}$ be $a$ prime ideal of $K_{2}$. We denote by $n$ a natural number. Then there exists an element $\gamma$ of $\mathfrak{D}_{K_{1}} \mathfrak{O}_{K_{2}}$ such that $\gamma \equiv 1\left(\bmod \mathfrak{P}^{n}\right)$ holds for all $\mathfrak{B}$ of $S$ above $\mathfrak{p}$ and $\gamma \equiv 0\left(\bmod \mathfrak{P}^{n}\right)$ for all $\mathfrak{\beta}$ of $S$ not above $\mathfrak{p}$.

Proof. We put

$$
S_{1}=\left\{\mathfrak{O}_{K_{1}} \mathcal{D}_{K_{2}} \cap \mathfrak{P}: \mathfrak{P} \in S, \mathfrak{P} \mid \mathfrak{p}\right\},
$$

and

$$
S_{2}=\left\{\mathfrak{D}_{K_{1}} \mathfrak{D}_{K_{2}} \cap \mathfrak{P}: \mathfrak{P} \in S, \mathfrak{P} \times \mathfrak{p}\right\} .
$$

Elements of $S_{1} \cup S_{2}$ are maximal ideals of $\mathfrak{S}_{K_{1}} \mathfrak{D}_{K_{2}}$. $S_{1} \cap S_{2}=\varnothing$ holds. So by Chinese remainder theorem there exists an element $\gamma$ of $\mathfrak{O}_{K_{1}} \mathfrak{O}_{K_{2}}$ such that $\gamma \equiv 1\left(\bmod \mathfrak{M}^{n}\right)$ for all $\mathfrak{M} \in S_{1}$ and $\gamma \equiv 0\left(\bmod \mathfrak{M}^{n}\right)$ for all $\mathfrak{M} \in S_{2}$ hold, which proves Lemma 1.

For a $\mathfrak{S}_{k}$-module $M$ and a prime ideal $p$ of $k$, we denote by $M_{p} S_{p}^{-1} M$, where $S_{p}$ is $\mathfrak{D}_{k}-p$. 
Lemma 2. Let $p$ be a prime ideal of $k$. Then there exist $\alpha, \beta \in K_{1} K_{2}$ such that

$$
\operatorname{ord}_{\mathfrak{\beta}} \alpha=\operatorname{ord}_{\mathfrak{\beta}} \beta=\operatorname{ord}_{\mathfrak{\beta}} \mathfrak{D}_{K_{1} K_{2}}\left(\mathfrak{O}_{K_{1}} \mathfrak{D}_{K_{2}}\right)^{*}
$$

hold for all prime ideals $\mathfrak{B}$ of $K_{1} K_{2}$ above $p$,

$$
\alpha\left(\mathfrak{D}_{K_{1}} \mathfrak{D}_{K_{2}}\right)_{p} \subset\left(\mathfrak{O}_{K_{1}} \mathfrak{O}_{K_{2}}\right)_{p}^{*} \subset \beta\left(\mathfrak{O}_{K_{1}} \mathfrak{D}_{K_{2}}\right)_{p}
$$

Proof. Firstly we prove the existence of $\alpha$ satisfying the condition. Let $\mathfrak{p}$ be a prime ideal of $K_{2}$ above $p$. Let $z$ be an element of $\mathfrak{O}_{K_{2}}$ with (iii) in 2.1. Since the dual module of $\mathfrak{D}_{k}[z]$ with respect to $\operatorname{Tr}_{K_{2} / k}$ is $f_{z}^{\prime}(z)^{-1} \mathfrak{O}_{k}[z]$, we have

$$
f_{z}^{\prime}(z)^{-1} \mathfrak{D}_{k}[z] \supset \mathfrak{D}_{K_{2} / k}^{-1}
$$

We take $d \in \mathfrak{D}_{K_{2} / k}$ which satisfies $\operatorname{ord}_{\mathfrak{q}} d=\operatorname{ord}_{\mathfrak{q}} \mathfrak{D}_{K_{2} / k}$ for all prime ideals $\mathfrak{q}$ of $K_{2}$ above $p$. Then we have

$$
d f_{z}^{\prime}(z)^{-1} \mathfrak{Z}_{k}[z]_{p} \supset \mathfrak{S}_{K_{2}, p}
$$

So we have

$$
d f_{z}^{\prime}(z)^{-1} \mathfrak{D}_{K_{1}}[z]_{p} \supset\left(\mathfrak{O}_{K_{1}} \mathfrak{O}_{K_{2}}\right)_{p} .
$$

By taking dual, we get

$$
g_{z}^{\prime}(z)^{-1} d^{-1} f_{z}^{\prime}(z) \mathfrak{O}_{K_{1}}[z]_{p} \subset\left(\mathfrak{D}_{K_{1}} \mathfrak{O}_{K_{2}}\right)_{p}^{*} .
$$

We put $\alpha_{\mathfrak{p}}=g_{z}^{\prime}(z)^{-1} d^{-1} f_{z}^{\prime}(z)$. We take $\gamma_{p}$ which satisfies the conditions in Lemma 1 , where $S$ is the set of all prime ideals of $K_{1} K_{2}$ above $p$ and $n$ is sufficiently large. We put

$$
\alpha=\sum_{p \mid p} \alpha_{p} \gamma_{p} .
$$

$\alpha$ satisfies the condition of Lemma 2. In fact, for a prime ideal $\mathfrak{B}$ of $K_{1} K_{2}$ and a prime ideal $\mathfrak{p}$ of $K_{2}$ with $\mathfrak{P}|\mathfrak{p}| p$, we have

$$
\operatorname{ord}_{\mathfrak{\beta}} \alpha=\operatorname{ord}_{\mathfrak{P}} \alpha_{\mathfrak{p}} \gamma_{\mathfrak{p}}=\operatorname{ord}_{\mathfrak{P}} \alpha_{\mathfrak{p}}=\operatorname{ord}_{\mathfrak{P}} \mathfrak{D}_{K_{1} K_{2}}\left(\mathfrak{D}_{K_{1}} \mathfrak{D}_{K_{2}}\right)^{*} \text {. }
$$

And clearly $\alpha$ satisfies (2), Lemma 2.

Secondly we prove the existence of $\beta$. For a prime ideal $\mathfrak{p}$ of $K_{2}$ above $p$ we take an element $z$ of $\mathfrak{D}_{K_{2}}$ with (iii) in 2.1. We put $\beta_{\mathfrak{p}}=g_{z}^{\prime}(z)$. By taking dual of $\mathfrak{D}_{K_{1}}[z] \subset \mathfrak{D}_{K_{1}} \mathfrak{D}_{K_{2}}$, we have

$$
\beta_{\mathfrak{p}}\left(\mathfrak{D}_{K_{1}} \mathfrak{D}_{K_{2}}\right) * \subset \mathfrak{D}_{K_{1}}[z]
$$


Therefore we have

$$
\left(\mathfrak{D}_{K_{1}} \mathfrak{O}_{K_{2}}\right) *\left(\sum_{\mathfrak{p} \mid p} \beta_{\mathfrak{p}} \mathfrak{O}_{K_{1}} \mathfrak{D}_{K_{2}}\right) \subset \mathfrak{D}_{K_{1}} \mathfrak{O}_{K_{2}}
$$

For a prime ideal $\mathfrak{p}$ of $K_{2}$ we take $\gamma_{\mathfrak{p}}$ satisfying the condition in Lemma 1 , where $S$ is the set of all prime ideals of $K_{1} K_{2}$ above $p$ and $n$ is sufficiently large. We put

$$
\beta=1 / \sum_{\mathfrak{p} \mid p} \beta_{\mathfrak{p}} \gamma_{\mathfrak{p}} .
$$

$\beta$ satisfies the conditions of Lemma 2. In fact, for a prime ideal $\mathfrak{P}$ of $K_{1} K_{2}$ and a prime ideal $\mathfrak{p}$ of $K_{2}$ with $\mathfrak{P}|\mathfrak{p}| p$, we have

$$
\operatorname{ord}_{\mathfrak{p}} \beta=-\operatorname{ord}_{\mathfrak{p}} \beta_{\mathfrak{p}} \gamma_{\mathfrak{p}}=-\operatorname{ord}_{\mathfrak{p}} \beta_{\mathfrak{p}}=\operatorname{ord}_{\mathfrak{p}} \mathfrak{D}_{K_{1} K_{2}}\left(\mathfrak{D}_{K_{1}} \mathfrak{O}_{K_{2}}\right)^{*} \text {. }
$$

And clearly $\beta$ satisfies (2), Lemma 2. Thus we proved Lemma 2.

Let $p$ be a prime ideal of $k$ and $\alpha, \beta$ elements of $K_{1} K_{2}$ satisfying (1), (2) in Lemma 2. Since

$$
\begin{aligned}
& {\left[\mathfrak{D}_{K_{1} K_{2}}\left(\mathfrak{D}_{K_{1}} \mathfrak{O}_{K_{2}}\right)_{p}^{*}: \alpha\left(\mathfrak{D}_{K_{1}} \mathfrak{O}_{K_{2}}\right)_{p}\right]_{\emptyset_{k, p}}} \\
& =\left[\mathfrak{O}_{K_{1} K_{2}}\left(\mathfrak{D}_{K_{1}} \mathfrak{O}_{K_{2}}\right)_{p}^{*}: \beta\left(\mathfrak{D}_{K_{1}} \mathfrak{O}_{K_{2}}\right)_{p}\right]_{\mathfrak{\wp}_{k, p}} \\
& =\left[\mathfrak{D}_{K_{1} K_{2}, p}:\left(\mathfrak{D}_{K_{1}} \mathfrak{O}_{K_{2}}\right)_{p}\right]_{\mathfrak{O}_{k, p}} \text {, }
\end{aligned}
$$

we have

$$
\alpha\left(\mathfrak{D}_{K_{1}} \mathfrak{O}_{K_{2}}\right)_{p}=\left(\mathfrak{O}_{K_{1}} \mathfrak{O}_{K_{2}}\right)_{p}^{*}=\beta\left(\mathfrak{D}_{K_{1}} \mathfrak{O}_{K_{2}}\right)_{p}
$$

So we have

$$
\begin{aligned}
& \left(\left[\left(\mathfrak{D}_{K_{1}} \mathfrak{O}_{K_{2}}\right)^{*}: \mathfrak{D}_{K_{1}} \mathfrak{O}_{K_{2}}\right]_{\bigcirc_{K_{1}}}\right)_{p}
\end{aligned}
$$

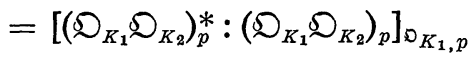

$$
\begin{aligned}
& =\left[\alpha\left(\mathfrak{D}_{K_{1}} \mathfrak{O}_{K_{2}}\right)_{p}:\left(\mathfrak{D}_{K_{1}} \mathfrak{O}_{K_{2}}\right)_{p}\right]_{\mathfrak{O}_{K_{1}, p}} \\
& =\mathrm{N}_{K_{1} K_{2} / K_{1}}\left(\alpha^{-1}\right) \mathfrak{\bigotimes}_{K_{1}, p} \\
& =\mathrm{N}_{K_{1} K_{2} / K_{1}}\left(\prod_{\sigma \neq 1} e_{\sigma \mid K_{2}}\right) \mathfrak{\bigcirc}_{K_{1}, p} \text {. }
\end{aligned}
$$

Hence we get

$$
\left(\left[\mathfrak{O}_{K_{1}} \mathfrak{O}_{K_{2}}\right)^{*}: \mathfrak{D}_{K_{1}} \mathfrak{O}_{K_{2}}\right]_{\mathfrak{O}_{K_{1}}}=\mathrm{N}_{K_{1} K_{2} / K_{1}}\left(\prod_{\sigma \neq 1} e_{\sigma \mid K_{2}}\right)
$$

\section{§3. Examples}

3.1. Let $k$ be a number field and $K$ its finite Galois extension with 
Galois group $G$. Let $K_{1}, K_{2}$ be intermediate fields of $K / k$. We denote by $H_{1}, H_{2}$ the subgroups of $G$ corresponding to $K_{1}, K_{2}$ respectively. We define $\sum_{K_{1}, K_{2}}$, a subset of $G$, by $H_{1} H_{2}-H_{1}-H_{2}$, where $H_{1} H_{2}$ is $\left\{h_{1} h_{2}: h_{1} \in H_{1}, h_{2} \in H_{2}\right\}$. Then the conductor $\mathfrak{f}_{K_{1}, K_{2}}$ of $\mathfrak{D}_{K_{1}} \mathfrak{D}_{K_{2}}$ with respect to $\mathfrak{D}_{K_{1} K_{2}}$ is $\prod_{\sigma \in \Sigma_{K_{1}, K_{2}}} e_{\sigma}$, where $e_{\sigma}$ is $\left(x-x^{\sigma}: x \in \mathfrak{D}_{K}\right)$. This can be proved by fundamental properties of elements. From this fact we know that $\mathfrak{D}_{K_{1} K_{2}}=\mathfrak{D}_{K_{1}} \mathfrak{D}_{K_{2}}$ holds if and only if for any prime ideal $\mathfrak{\beta}$ of $K$ and any $\sigma \in \sum_{K_{1}, K_{2}}, \sigma$ is not contained in the inertia group of $\mathfrak{P}$.

3.2. We give some examples.

1. $G=\operatorname{Gal}(K / k)=\left\langle\sigma, \tau: \sigma^{2}=\tau^{2}=(\sigma \tau)^{2}=1\right\rangle$.

Let $K_{1}, K_{2}, K_{3}$ be the fixed fields of $\langle\sigma\rangle,\langle\tau\rangle,\langle\sigma \tau\rangle$ respectively. Then we have

$$
\begin{aligned}
\sum_{K_{1}, K_{2}} & =\{\sigma \tau\}, \\
\mathfrak{f}_{K_{1}, K_{2}} & =e_{\sigma \tau}=\mathfrak{D}_{K / K_{3}} .
\end{aligned}
$$

$\mathfrak{D}_{K}=\mathfrak{D}_{K_{1}} \mathfrak{D}_{K_{2}}$ holds if and only if $K / K_{3}$ is unramified.

2. $G=\operatorname{Gal}(K / k)=\left\langle\sigma, \tau: \sigma^{3}=\tau^{2}=1, \tau^{-1} \sigma \tau=\sigma^{-1}\right\rangle$.

Let $K_{i}$ be the fixed field of $\left\langle\tau \sigma^{i-1}\right\rangle(i=1,2,3)$ and $M$ the fixed field of $\langle\sigma\rangle$. 3.2.1 $\sum_{K_{1}, K_{2}}=\{\sigma\}$,

$$
\mathfrak{f}_{K_{1}, K_{2}}=\mathfrak{e}_{\sigma}=\mathfrak{D}_{K / M}^{1 / 2} .
$$

$\mathfrak{D}_{K}=\mathfrak{D}_{K_{1}} \mathfrak{D}_{K_{2}}$ holds if and only if $K / M$ is unramified.

3.2.2 $\sum_{K_{1}, M}=\left\{\tau \sigma, \tau \sigma^{2}\right\}$,

$$
\mathfrak{f}_{K_{1}, M}=e_{\tau \sigma} e_{\tau \sigma^{2}}=\mathfrak{D}_{K / K_{2}} \mathfrak{D}_{K / K_{3}} .
$$

$\mathfrak{D}_{K}=\mathfrak{D}_{K_{1}} \mathfrak{O}_{M}$. holds if and only if $M / k$ is unramified.

3. $G=\operatorname{Gal}(K / k)=A_{4} \longrightarrow \operatorname{Aut}(\{a, b, c, d\})$.

We put $x=(a b)(c d), y=(a c)(b d), z=(a d)(b c), t=(a b c)$ and $H=$ $\{1, x, y, z\}$. Let $K_{1}, K_{2}, K_{3}, K_{4}$ be the fixed fields of $\langle t\rangle,\langle t x\rangle,\langle t y\rangle,\langle t z\rangle$ respectively. Let $L_{1}, L_{2}, L_{3}$ be the fixed fields of $\langle x\rangle,\langle y\rangle,\langle z\rangle$ respectively. Let. $M$ be the fixed field of $H$.

3.3.1 $\sum_{K_{1}, M}=\left\{t^{2} x, t^{2} y, t^{2} z, t x, t y, t z\right\}$.

$$
\mathfrak{f}_{K_{1}, M}=\mathfrak{D}_{K / K_{2}} \mathfrak{D}_{K / K_{3}} \mathfrak{P}_{K / K_{4}} \text {. }
$$

$\mathfrak{D}_{K}=\mathfrak{D}_{K_{1}} \mathfrak{O}_{M}$ holds if and only if $M / k$ is unramified.

3.3.2 $\sum_{K_{1}, K_{2}}=\left\{x, t z, t^{2} x, z\right\}$, 


$$
\mathfrak{f}_{K_{1}, K_{2}}=\mathfrak{D}_{K / L_{1}} \mathfrak{P}_{K / L_{3}} \mathfrak{D}_{K / K_{3}}^{1 / 2} \mathfrak{P}_{K / K_{4}}^{1 / 2} \text {. }
$$

$\mathfrak{S}_{K}=\mathfrak{D}_{K_{1}} \mathfrak{D}_{K_{2}}$ holds if and only if $K / k$ is unramified.

3.3.3 $\sum_{K_{1}, L_{2}}=\left\{t^{2} y, t y\right\}$,

$$
\mathfrak{f}_{K_{1}, L_{2}}=\mathfrak{D}_{K / K_{3}}^{1 / 2} \mathfrak{D}_{K / K_{4}}^{1 / 2} .
$$

$\mathfrak{D}_{K}=\mathfrak{D}_{K_{1}} \mathfrak{D}_{L_{2}}$ holds if and only if $M / k$ is unramified.

\section{REFERENCES}

[1] A. Fröhlich, Local fields, Chapter 1 in "Algebraic Number Theory", Proceeding of the Brighton Conference, London and New York, 1967.

[2] G. Shimura, Construction of class fields and zeta functions of algebraic curves, Ann. of Math., 85 (1967), 58-159.

University of Tokyo 